\title{
Pollen parent transfer mitochondria to offspring
}

Biryukov M. ${ }^{1 *}$, Blinov A.G. ${ }^{1,2}$, Sokolov V.A. ${ }^{2}$

${ }^{1}$ Institute of Cytology and Genetics, SB RAS, Novosibirsk, Russia

${ }^{2}$ Institute of Molecular and Cellular Biology, SB RAS, Novosibirsk, Russia

*e-mail: birykov@bionet.nsc.ru

In plant cells, DNA is located in three compartments: nucleus, plastids and mitochondria. Genes transmitted by chromosomes of the nucleus inherited by the offspring of Mendel. Plastids and mitochondria can be inherited from the mother, from the father, or from both parents. In most cases, the plastids are transmitted by the parent, since they are either not included in the cells of the generative sperm, or remain fertilized during fertilization. Only under experimental conditions was it possible to obtain plastid transmission from Nicotiana to the offspring with a frequency of $10^{-4}$ to $10^{-5}$. Maternal inheritance of mitDNA in plants has several important consequences for its evolutionary dynamics. First of all, such a transfer determines the direct contribution of maternal mitDNA to fitness determined by the number and quality of the seeds produced. Transmission only on the maternal line is of particular importance for the formation of the population structure of plants, especially those in which pollen spreads over long distances. For a long time it was believed that the mitochondrial genomes of plants are transmitted to the offspring strictly from the mother parent. At the same time, such a method of their inheritance is based on the use of a number of special, finely organized mechanisms that ensure its rather strict functioning. However, there are cases of transmission of mitDNA from fathers, and it is possible that this phenomenon occurs quite often. In this communication, we analyzed the transfer of genetic information in hybrids of two Zea mays lines (B73 and Mo17) and Tripsacum dactyloides line by nuclear, chloroplast and mitochondrial genomes. To this end, partial nucleotide sequences of the nuclear Pox3 gene, the chloroplast trnL gene and two mitochondrial genes (S-male sterility locus and Nad4), in the original parental species and F1 hybrids have been established. Based on the molecular phylogenetic analysis, it was found that the nuclear genome in hybrids comes from both parents, the chloroplast is transmitted exclusively on the maternal line, and the mitochondrial genome can be obtained both from the maternal and the paternal lines. 